\title{
Resolving the Asymmetry of On-Exit versus On-Entry in Executable Models of Behaviour
}

\author{
Vladimir Estivill-Castro and René Hexel \\ School of ICT, Griffith University, Nathan Campus, Nathan 4111, Australia
}

Keywords: Executable UML, UML State Charts, Model-based Testing and Validation, Modelling Behaviour.

Abstract: $\quad$ For the UML, state charts are by far the most used modelling tools, both to communicate behaviour and to produce executable models. We investigate the inherent asymmetry of On-Entry and On-Exit Actions in UML Statecharts. We show first that the apparently simple and symmetric rules for handling the sequencing of On-Entry and On-Exit actions are hard to fully comprehend and apply effectively by software developers. Second, defining a semantics that results in executable models for applications such as reactive-systems and real-time systems is very delicate. Third, formal verification can be hampered because the semantics results in a combinatorial explosion of states. We evaluate the understandability of the semantics by taking out experiments with various tasks comprising sample UML Statechart and logic-labelled finite state machines (LLFSMs). Several experiments with software developers enable us to dissect how issues of understandability of state diagrams relate to nesting or event-driven vs logic-labelled. Since logic-labelled finite state machines achieve model composition through a subsumption architecture (suspend/restart/resume) we propose a specific alternative semantics for logic-labelled finite state machines that is suitable for robotic and embedded systems.

\section{INTRODUCTION}

Wikipedia is the source of information for billions of people on the planet. Its English version records more than 20 billion views per month. Wikipedia's page on UML state machines ${ }^{1}$ declares that "every state in a UML state chart can have optional entry actions, which are executed upon entry to a state, as well as optional exit actions, which are executed upon exit from a state. Entry and exit actions are associated with states, not transitions. Regardless of how a state is entered or exited, all its entry and exit actions will be executed." Moreover, the implication is that the On-Entry and On-Exit sections of a state are shorthand notation for what otherwise would be the cumbersome inclusion of more states. Even more importantly, they are treated symmetrically as set-up and tear-down phases: The value of entry and exit actions is that they provide means for guaranteed initialization and cleanup, very much like class constructors and destructors in Object-oriented programming." Thus, one can assume that the On-Entry and On-Exit sections are some form of notational simplification for potential pre-states and post-states (see

${ }_{1}^{1}$ en.wikipedia.org/wiki/UML_state_machine
Figure 1). The situation is not much different with fUML (a subset of the standard Unified Modelling Language (UML) for which standard, precise execution semantics are provided). Although fUML uses Clause 15 of the UML Superstructure to define the execution semantics for statecharts, it describes the entry behaviour and the exit behaviour of a state in completely symmetric terms when it describes Alf (Object Management Group, 2017, Page 328). We argue here that there are profound implications for the semantics of these UML forms, both for understandability of UML diagrams and for correctness, validation and formal verification of executable models. Among practitioners, a top criterion for the adoption of UML's representation is whether such representations are understandable (Petre, 2013). Professional software developers' largest gripes are around complexity and lack of formal semantics (Petre, 2013).

Little would be achieved without some form of model composition. The subsumption architecture enables layers of timed logic-labelled finite-sate machines to structure more sophisticated behaviours on top of simpler behaviours (Brooks, 1986). The UML proposes orthogonal regions (Samek, 2008, Chapter 2) (so-called AND-decomposition, which also implies concurrency) and nesting sub-states (so-called 


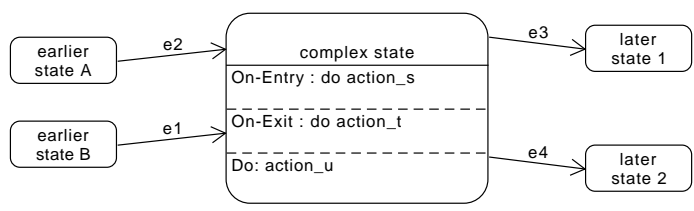

(a) Typical state with On-Entry and On-Exit sections.

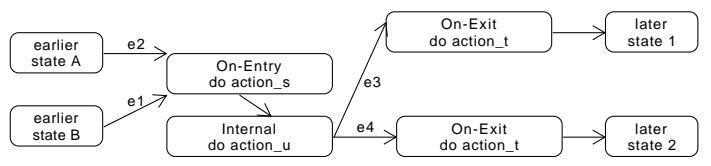

(b) Equivalent On-Entry and On-Exit behaviour.

Figure 1: On-Entry and On-Exit as intermediate states.

OR-decomposition). Nested states have been labelled "great diagrammatic simplifications when a set of events applies to several sub-states" (Douglass, 1999) Some (Samek, 2008, Page 69) regard hierarchically nested states by Harel and Politi (1998), as the most important invention. However, Mellor (2000) noted several issues and complex semantic implications. Similarly to the Ultimate Hook Pattern, nested states are a mechanism to produce common facilities and simplification to event-handling policies. The sharing of behaviour through a notation that implies inheritance is very powerful and widely used in many aspects of object-orientation and the UML in particular. This abstraction capacity mostly follows Liskov's Substitution Principle (Liskov and Wing, 1994) and implies that a sub-state of a composite state has behavioural inheritance. However, in the case of states, the is a relationship of inheritance is replaced by is in (is-in-a-state) relationship (Samek, 2008, Page 72). Consider Figure $2 .^{2}$ Here, the system in the toasting state $i s$-in the heating state.

We have carried out a series of experiments on the understandability of two particular issues in UML diagrams. First, the asymmetry of On-Entry actions versus On-Exit actions (which is already present in Figure 1, because On-Exit actions require an additional implicit state per transition). Second, the complexity of state-nesting. Our results are complementary to several of studies on the understandability of UML state charts, but we stress here the theoretical and experimental validation of metrics (Genero et al., 2003) such as NEntryA (number of entry actions), NExitA (number of exit actions), and NCS (number of composite states). In previous work, these

three metrics (NEntryA, NExitA, and NCS), although theoretically validated and inspired by the lit-

\footnotetext{
${ }^{2}$ Figure 2 (Samek, 2008, Figure 2.7) appears in Wikipedia's page on UML state machines and is distributed as commons material; we also used it on our experiments.
}

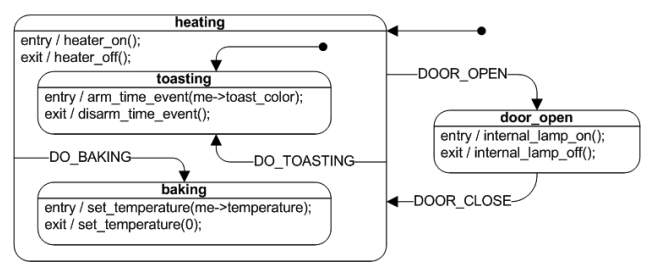

Figure 2: A sample UML state chart.

erature, have experimentally been shown not to be correlated with the understandability of UML diagrams (Genero et al., 2003). Our results indicate that NEntryA (number of entry actions) and NExitA (number of exit actions) are indeed relevant for understandability of a state diagram. The first set of metrics experimentally correlated to the understandability of UML state diagrams were NA (number of activities), NSS (number of simple states), NT (number of transitions), and NG (number of guards) (Genero et al., 2003). However, as the results were non-conclusive, further research followed (Cruz-Lemus et al., 2005, 2009). We still believe that those other metrics are relevant to the understandability of UML state diagrams. However, we emphasise new aspects, the asymmetry of the On-Entry and the On-Exit actions, the eventdriven nature of UML vs the deterministic execution of logic-labelled finite-state machines (LLFSMs), and naturally the nesting mechanism for composition. We note again that, although abstraction and understandability had been heralded for nesting states, this was not so evident in experimental settings (Cruz-Lemus et al., 2005, 2009). Our point here is that the issues of nesting, and the asymmetry of On-Exit versus OnEntry have been neglected from detailed analysis.

We show that the apparently simple and symmetric rules for handling the sequencing of On-Entry and On-Exit actions represent a series of fallacies. First, these rules are very hard to comprehend and to apply by software developers, especially when timing issues and composition are involved. Second, defining a semantics that results in executable models for applications such as reactive systems and real-time systems is very delicate. Third, verification is hampered because the semantics results in a combinatorial explosion of states. We derive these conclusions from several experiments with software developers and dissect how issues of understandability of state diagrams relate to nesting, or event-driven vs logic-labelled. Since LLFSMs achieve model composition through a subsumption architecture (suspend/restart/resume) we propose a specific alternative semantics for LLFSMs that is suitable for robotic and embedded systems.

UML state charts are ubiquitous and it has been documented they, along with class diagrams, are one of the top used artefacts (Reggio et al., 2013). They 
have very strong penetration in embedded systems, and are even used as executable models, synthesising VHDL (Wood et al., 2008). Nevertheless, UML state charts remain the subject of strong criticism (Rumpe, 2002; Börger et al., 2000; Glinz, 2000; Reggio et al., 2000). On one hand, the criticism is accurate regarding the ambiguous semantics, but on the other hand, UML is best used at the conceptual level. In any case, we found usability studies rarely support such criticism. The issue of understandability is subject of some studies we review in the next section.

\section{UNDERSTANDABILITY}

How useful is UML? Experimental studies have confirmed that visual and textual standard notations enable communicating software designs to stake holders. Moreover, UML can be cost-effective, as shown by Dzidek et al. (2008), who evaluated the cost of using UML at a degree of formality that is realistic for being a reflection of the running code. The study involved 20 senior developers (10 with UML experience) on five realistic maintenance tasks that required between 1 to 2 weeks. As a secondary goal, this study identified plausible and necessary conditions for UML to be effective. One of the assets of the study was that subjects were experienced developers. Nevertheless, a one day UML refresher was offered to all participants to level the experience. The study only considered class diagrams and sequence diagrams and concluded that expertise with UML and usability of associated tools are strong influencing factors in the cost-effectiveness of using UML.

Along the same lines are studies on UML's usecase templates. Mustafa (2010) discusses the importance of the human dimensions in understandability of modelling UML artefacts, as these are the main communication vehicle between all stake holders, including developers. Once again, the usability is linked to simplicity; UML artefacts must be intuitive to understand to be successful (Mustafa, 2010). Although focussed on use-case templates and diagrams, Mustafa (2010) reviewed previous UML comprehension studies, noting a focus on class diagrams (structural descriptions), sequence diagrams, and stereotypes.

UML diagram understandability is related to cognitive load; and thus, inexperienced users struggle with diagrams that require heavy, intrinsic cognitive load correlating with diagram details (Mustafa, 2010). Mustafa (2010) used the following definition of understandable: "The use case model is understandable if it allows users to recognise problem domain information and extend their understanding in problem solving." Therefore, Mustafa (2010) recommends that for evaluating understandability, besides question accuracy, experimenters shall evaluate the time required to answer the question. "understanding is a cognitive process, [and] it is difficult to directly observe it, and tests to measure participants' performance were conducted to assess the level of understanding cognitively developed by each participant." (Mustafa, 2010).

Understandability of UML state charts has been the focus of Cruz-Lemus et al. (2005, 2009). The research on the comprehension of state charts commenced with theoretical and experimental studies of the features that raise interpretation difficulty (Genero et al., 2003). Composite states add comprehensibility when users have prior familiarity with their features (Cruz-Lemus et al., 2005), but counterintuitively, no direct link between composite state complexity and UML state chart understandability was established. The hypothesis is that the use of composite states provides simplification, and then enhances understandability; but this hypothesis is not true for inexperienced users (Cruz-Lemus et al., 2005).

We hypothesise that users find UML state charts hard to understand because their nesting usually implies uncontrolled concurrency. That is, nesting of states, although simply described (see Wikipedia quote earlier), implies complex rules to resolve the sequence of execution. As such, we believe that logiclabelled finite-state machines (LLFSMs) are more understandable. There is some justification for this hypothesis. LLFSMs execute under a deterministic schedule. Previous work with formal verification and model checking with LLFSMs (Estivill-Castro et al., 2012) demonstrates that LLFSMs avoid the exponential explosion of associated Kripke structures used as input to model checkers. By contrast, UML state charts are event-driven, requiring a complex event handling process of at least five sub-steps.

Event Generation: All generated events must be channelled to those state charts whose transitions wait for the event.

Event Conveyance: Events are transported to current objects and states, theoretically with no delay and without changing the event.

Event Reception: Events are placed on queues, typically one queue per state chart (Samek, 2008).

Event Dispatch: The current event is de-queued, activating all responders as per the Run-UntilCompletion semantics (Samek, 2008).

Event Consumption: Indicates that the event has been handled; in some cases, removal from the queue is just part of this step (Samek, 2008). 
This mechanism implies call-backs associated with the corresponding events. As the fundamental aspect of the Hollywood principle, it is often viewed favourably and used in many software patterns to minimise coupling. However, callback order of execution becomes unpredictable, requiring a model checker to evaluate all paths of execution. Formal verification must consider all possible orders in which events may be queued, despite such combinatorial explosion. The semantics of hierarchically nesting of states in UML is commonly specified with an interlingua approach. Namely, a construct is considered a shorthand notation for a larger construct in the language or another language for which the semantics has been provided. For hierarchically nesting, "the Cartesian product machine is used as the interlingua semantics of state charts" (Drusinsky, 2006, Page 63). Moreover, "an event can trigger a transition in all active threads, in some action threads, or in none" (Drusinsky, 2006, Page 63). UML users must keep in mind all aspects of the event-driven Run-Until-Completion semantics and the nested state interlingua semantics (cognitive load).

By comparison, LLFSMs use two fundamental approaches as key composition mechanisms.

\section{Status/Control Message Passing: Orthogonal}

behaviours with different responsibilities can synchronise through a shared memory reader/writer architecture that avoids race conditions.

Using Mechanisms to suspend/resume/restart: Enabling all sorts of machine hierarchies; in particular, allowing subsumption architectures.

In this paper, we experimentally evaluate the hypothesis proposed.

\section{METHOD}

We performed several controlled experiments and their replication under Griffith University Ethics Reference Number: 2018/785. Subjects with software development and software modelling experience were either 1) randomly divided into two or three groups for different tasks or 2) observed with respect to two or three different features on the same task. We always recorded the time to complete the task.

Our experiments consisted of formulating a hypothesis, such as "use of composite states improves understandability of UML" and then used a measure that considers how quickly subjects solve a task and how accurately they solve the task. The measure we used is "understandability efficiency" (Cruz-Lemus et al., 2005) as the accuracy (the number of correct answers) divided by the time taken. We completed the experimental formulation by defining a hypothesis testing scenario; for example:

$H_{0}$ : the use of composite state diagrams does not improve the understandability efficiency.

$H_{1}$ : the use of composite state diagrams does improve the understandability efficiency.

\subsection{Experimental Process}

The experiments took place in Queensland, Australia, during July and August 2018. The subjects were students of Griffith University from two campuses and four different degrees (one master and three undergraduate programs). Besides subjects being students, other aspects in common with earlier UML understandability studies (Cruz-Lemus et al., 2005; De Lucia et al., 2010; Genero et al., 2003) were as follows.

1. The tasks in the experiment did not require a high level of industrial experience.

2. Students were in their final year, completing a course in software engineering, or they were masters students who had already completed a prior IT degree.

3. Subjects received significant instruction on the main constructs of the UML. Model-Driven development was illustrated and exercised in laboratories using ARGO-UML (Robbins, 1999).

4. Students developed UML class diagrams and generated code in C++, Java and SQL, analysing multiple aspects of the mappings.

5. State charts were used in laboratories using model-driven development and executable models through the $Q M^{\mathrm{TM}}$ tool. Students were required to review "A Crash Course in UML State Machines" distributed by Quantum ${ }^{\circledR} \mathrm{L}^{\mathrm{e}} \mathrm{aPs}^{3}$ and based on (Samek, 2008, Chapter 2).

6. Subjects, therefore, had similar expertise as in the literature and were given similar motivation, such as explaining that the tasks would be illustrative of the final exam (Cruz-Lemus et al., 2005; De Lucia et al., 2010; Genero et al., 2003).

7. As part of the experimental procedure, prior to attempting the tasks, subjects were given the opportunity to review material on UML state charts, e.g. the above Wikipedia page, plus two others ${ }^{4}$.

\footnotetext{
${ }^{3}$ https://www.state-machine.comstate-machine.com

${ }^{4}$ https://www.uml-diagrams.org/state-machine-diagra ms.htmland https://www.lucidchart.com/pages/uml-statemachine-diagram
} 
In experimental software engineering, when subjects face relatively small tasks, only minor differences between professionals and students are expected (Basili et al., 1999; Höst et al., 2000). Thus, we also adopt the common assumption that our students as subjects are appropriate (Cruz-Lemus et al., 2005; De Lucia et al., 2010; Genero et al., 2003).

\subsection{Experimental Design}

We conducted several of two types of experiments in different weeks, with voluntary participation.

Same Treatment of All Subjects: Evaluation of two or more aspects of the subject's performance. Everyone solved the same problem, then a correlation analysis was performed. E.g., all subjects are to describe all diagram features and we measure the accuracy of describing On-Entry behaviour versus describing On-Exit behaviour.

Different Treatments of Subjects: All subjects answered the same questions about different, randomly assigned diagrams, and an ANOVA (or $t$ test, if two classes) was performed. Groups were randomly assigned, and all were asked to complete the same task; however, each group proceeded with diagrams with a specific feature (for example state nesting) with the control from a diagram without the feature.

\subsection{Experimental Tasks}

All tasks consisted of the interpretation of simple UML diagrams (comprising of at most three levels of nesting, typically two, and at most one machine and one sub-machine). Typically, the notation had been presented earlier. For models that produced short output, subjects were asked to anticipate the output generated. For behaviours that generated continuous output, subjects were required to identify the main traits of the behaviour, or alternatively subjects we asked whether a particular sequence of output statements occurred in that precise order.

Experiments were conducted as extra-laboratory exercises within the context of a third-year Software Engineering course. Responses were anonymous; thus, students were not evaluated on their individual performance. We followed De Lucia et al. (2010) in selecting easy to comprehend data models. The subjects were not aware of any of our scoring approaches or metrics of interest, and they were allowed plenty of time to complete the task. We emphasised the premise that a simple data model was preferred over a more complex one (as the focus was not the application domain nor the accuracy with which the model reflects complex situations). The attention of our research is the notation used to represent the diagram (De Lucia et al., 2010). We also emphasise that for understanding tasks (and the understandability of UML artefacts) it is common to request subjects to provide as much information as possible and to define the expected response prior to issuing the task.

\subsubsection{Calibration}

Our first experimental task was the verbatim reuse of the original Questionnaire of Appendix A by CruzLemus et al. (2005), and randomly partitioning the subjects to answer the Questionnaire with respect to the original Figures 5 (F5) and 6 (F6) (Cruz-Lemus et al., 2005), We remark that the task could be considered particularly simple, and unfortunately (CruzLemus et al., 2005, Figure 5) or (Cruz-Lemus et al., 2005, Figure 6) may be translations from Spanish to English (the figures have a spelling error). Each figure is supposed to have an equivalent UML diagram that models the same behaviour of a phone call: F5 uses nesting states while F6 has no nested states. However, F6 is a simplified version of F5 (recall the interlingua semantics of nested states). That is, F6 draws much fewer transitions than those implied by F5. This difference demonstrates that indeed, nesting exposes many transitions that, if drawn, would clutter the diagram (perhaps unnecessarily so).

Importantly, the figures are not semantically equivalent. Nevertheless, the questions in the Questionnaire (Cruz-Lemus et al., 2005, Appendix A) do not explore this issue.

\subsubsection{Simple, Nested Model}

Our second experimental task consisted of predicting the output of a simple model, in particular, to comprehensively describe the information provided by the UML state chart in Figure 2. For this task, the prepared answer was to obtain paragraphs equivalent to those in Figure 3. Also a questionnaire that had 8 questions testing subjects on whether they could correctly identify behaviour (sequencing) on On-Exit conditions, On-Entry conditions, On-Entry and OnExit conditions, Run-Until-Completion, State Nesting, ordering of events, ordering of nesting (priority on exiting a hierarchy of nested states), and reentering a hierarchy of nested states.

\subsubsection{Non-nested LLFSM}

In the third task, we used the downloadable version ${ }^{5}$ of the clfsm scheduler for logic-label finite-state ma-

\footnotetext{
${ }^{5}$ www.mipal.net.au/downloads.php
} 


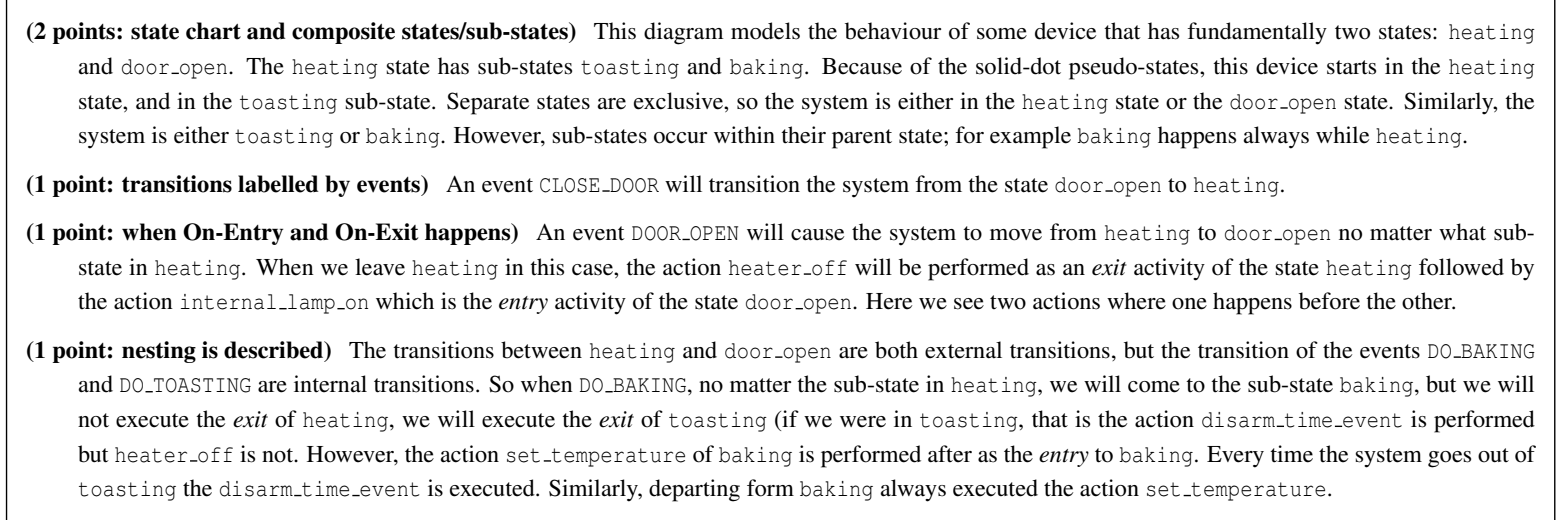

Figure 3: Expected point we believe should not be lost in translation where interpreting the model of Figure 2 in English.

chines and the MiEdit editor under Ubuntu 16.04 and ROS-Kinetic (we did not implement the message passing approach for communicating LLFSMs, but used rostopics and rosservices from ROS' middleware). Subjects were provided practice in executing LLFSMs with the clfsm scheduler.

For the task, subjects were required to predict the output of the logic-labelled finite-state machine in Figure 4. This apparently simple model has implications for understanding the notion of guards, when in a ringlet is a transition evaluated, and whether the On-Exit is executed in a final state.

\subsubsection{Nested LLFSMs}

For the fourth task, the setting again was the executable model defined by an LLFSMs and the environment was the clfsm scheduler and communication with ROS-Kinetic middleware. In preparation for this task, the clfsm scheduler capabilities to suspend/resume/restart one LLFSM from another LLFSM were demonstrated. Although the executable model produces continuous output, the task consisted of formulating a qualitative prediction of the execution of the LLFSM in Figure 5.

\subsubsection{Subsumption and Delegation}

For the preparation of the fifth task, we provided examples of message passing using the ROS publisher/subscriber software pattern (rostopics) and the client/server software pattern (rosservices). Because LLFSMs do not use callbacks, instructions were provided on wrapping signals (events) in a rostopic into a rosservice (as illustrated by the third example of the downloadable ROS LLFSM examples for ROS on the above download site).

In this task, subjects were required to implement the model presented in Figure 2 using LLFSMs; in particular, the behaviour that responds to the signals to bake and to toast; then to construct a second behaviour that responds to the signals open door and close door, and finally to integrate the two behaviours. Subjects were required to commit to one of the two strategies by which LLFSMs represent nesting of states: that is, subjects were asked to chose between suspend/resume/restart or to use a delegation (forwarding) of messages.

\subsubsection{Randomised Diagrams}

In the sixth and final experiment, subjects were randomly partitioned into three groups and provided the same Questionnaire (Figure 6). However, each group was provided with a different diagram. The first group was provided the diagram in Figure 2, while the second was provided with a model solution to its implementation using suspend/resume/restart with LLFSMs, the third was provided with a model solution using delegation/forwarding with LLFSMs.

\subsection{Replication}

All experiments were replicated between two campuses with common offerings of the same course, but different staff (lecturers, instructors, and tutors). Also, each campus had an additional, specific program not offered at the other (Computer Science and Software Engineering), with some overlap (IT).

\section{RESULTS}

We now report on the experiments described earlier and detail the perception and understandability of the different models. 


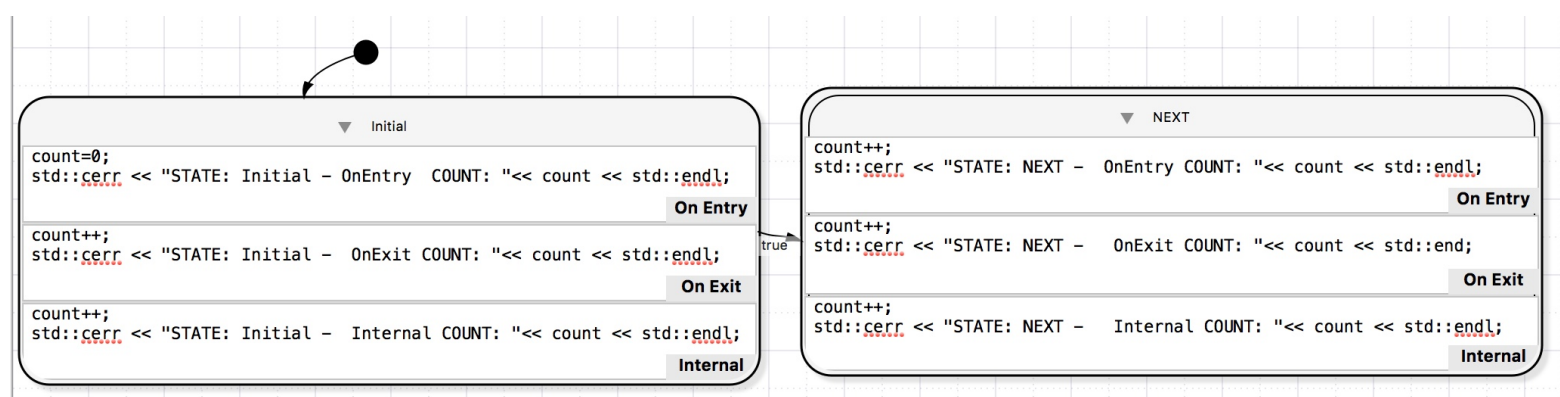

Figure 4: A simple logic-labelled finite-state machine.

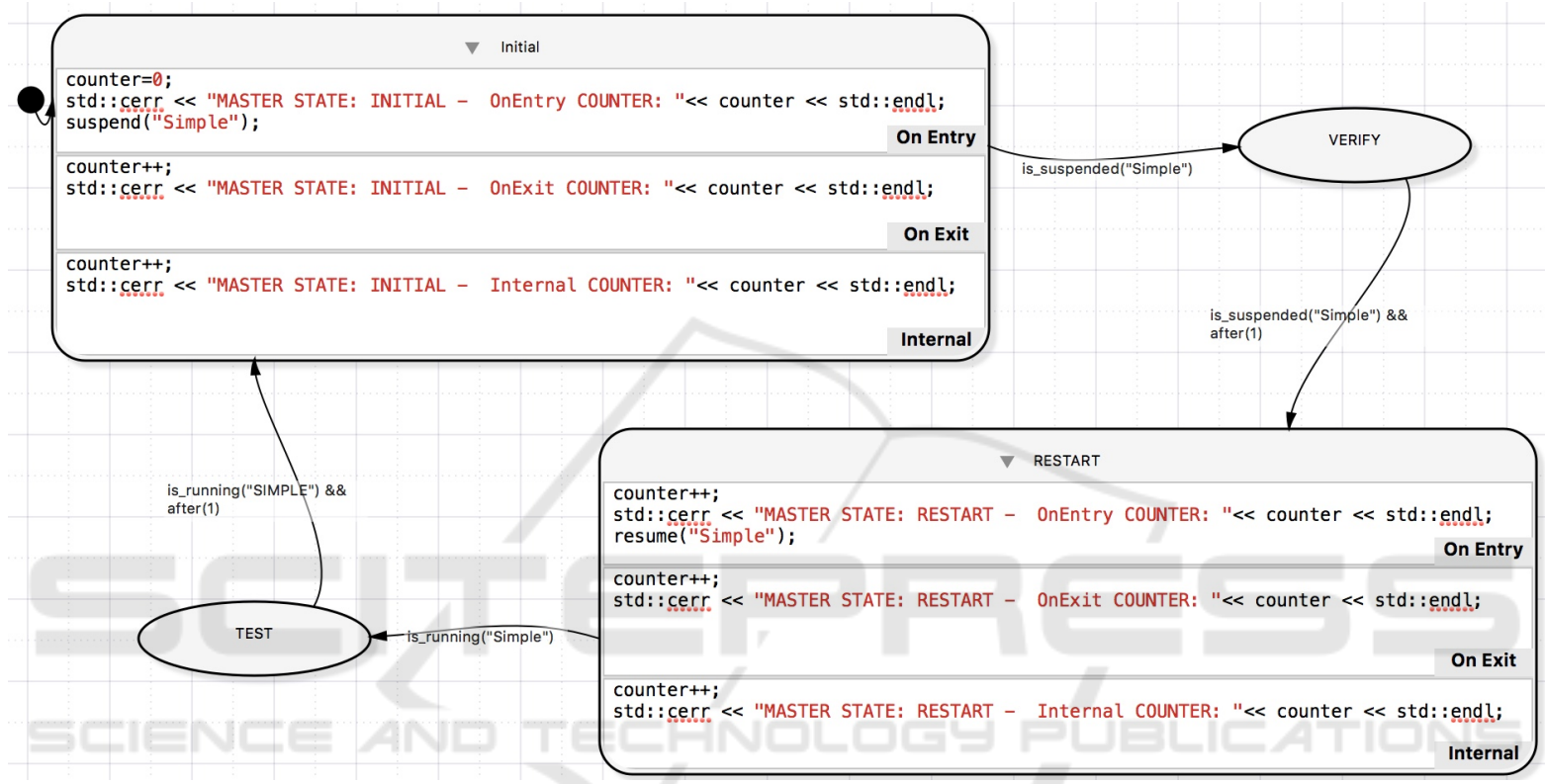

Figure 5: A logic-labelled finite-state machine that affects another LLFSM.

The diagram models the behaviour of a toaster oven. Assume that no events have been issued prior to each of the questions below, and that the two behaviours were launched concurrently in the order Outer followed by Inner. Answer only in terms of the actions: heater_on(), heater_off (), arm_time_event (me->toast_color), disarm_time_event (), set_temperature (me->Temperature), set_temperature (0), internal_lamp_on (), and

internal_lamp_off ()

1. If the event to DO_BAKING is received, what is/are a/the sequence of actions produced by all behaviours involved?

2. If the event to DO_BAKING is received; and later, after a few seconds, the event to DOOR_OPEN happens, what is/are a/the sequence of actions produced by all behaviours involved?

3. If the event to DO_BAKING is received, and while the action disarm_time_event ( ) is being performed, the DOOR_OPEN happens, what is/are a/the sequence of actions produced by all behaviours involved?

4. If the event to DO_BAKING is received, and while the action set_temperature (me->Temperature) is running the DOOR_OPEN happens, what is/are a/the sequence of actions produced by all behaviours involved?

5. If the event to DO_BAKING is received, and after a few seconds the event to DOOR_OPEN happens, and while the action set_temperature $(0)$ is being performed, the DOOR_CLOSE happens, what is/are a/the sequence of actions produced by all behaviours involved?

6. If the event to DO_BAKING is received, and after a few seconds the event to DOOR_OPEN happens, and while the action heater_off () is executing, the DOOR_CLOSE happens, what is/are a/the sequence of actions produced by all behaviours involved?

7. Write down the minimum sequence of events and conditions needed, to go from the state toasting to the state baking, and back to toasting, but this going back is not caused by an event to DO_TOASTING:

8. Write down the minimum sequence of events and conditions needed, to go from the state toasting to the state baking, and back to toasting.

Figure 6: Questionnaire related to equivalent diagram and including Figure 2. 


\subsection{Calibration}

The replication of the Questionnaire by Cruz-Lemus et al. (2005), Appendix A, shows no evidence of a difference between nested and plain diagrams. Our experimental outcome is equivalent to the results CruzLemus et al. (2005) reported. On the two campuses we had 18 and 20 subjects respectively, each equally divided into the two groups (nested versus plain). This calibration enabled us to judge the understandability efficiency. There seems to be no significant improvement (or difference) in understandability/efficiency by using nested states. We offer here a new explanation derived from our earlier observations and still congruent with the original conclusions by Cruz-Lemus et al. (2005). Nested states are an advanced concept. No-one would use introductory state charts with nested states (not even the earliermentioned crash course on UML state charts does such a thing), as nested states represent a significant cognitive load (and indeed an innovation and contribution by Harel, as also mentioned before). But users reach command of this artefact when experience and regular usage assimilates the implicit semantics that the interlingua semantics implies. Users (in the most Piagetian constructivist style) must form and adapt their conceptual frameworks to efficiently operate the potential combinations implied by nested states.

\subsection{Simple, Nested Model}

Our first remarkable result is the score difference observed for subjects answering questions regarding On-Exit actions vs On-Entry actions on the same diagram. Our questionnaires had 8 questions: a correct answer provided one point, an incorrect resulted in a negative point. For each subject, we subtracted their score for the On-Exit answer from the score for the On-Entry answers. The null hypothesis was that the mean of these differences is 0 . For our first campus experiment, with $N=51$ respondents, the mean of the difference scores was 3.00 with a standard deviation of 3.85. The standard error of the mean was 0.54. A $t$-test with 50 degrees of freedom rejects the null hypothesis ( $p$-value less than 0.00001$)$. The replication at the second campus had only $N=26$ respondents; nevertheless, the mean of the score differences was 2.11, with a standard deviation of 3.97. This results in an estimate of the standard error for the mean of 0.41 and the $t$-test with 25 degrees of freedom also rejects the null hypothesis ( $p$-value less than 0.00001). Thus, our experimentation reveals that subjects have different capacity to answer symmetrical questions regarding On-Entry sections of state charts as opposed to On-Exit sections. The mean accuracy is higher for the On-Entry questions than the On-Exit questions.

\subsection{Non-nested LLFSM}

The notion of logic-labelled finite-state machines (LLFSMs) could be seen as UML models with no events and only guards. Drusinsky (2006) considers LLFSMs under the name of procedural state machines, and he acknowledges that in that case, the model is not at the mercy of the arrival of events: "because [the automaton] can access the input symbols at any time, it can visit states as fast as we wish" (Drusinsky, 2006, Page 15).

Note that the notion of guard is typically introduced with a discussion that UML state charts are extended state machines (Samek, 2008, Chapter 2). In LLFSMs, since there are no events, exactly when the Boolean condition is evaluated is an important aspect of their precise semantics (a snapshot of all external variables is taken before commencing of a ringlet, and all guards of all transitions are evaluated in this context). For UML, this issue is somewhat ambiguous, the expressions are meant to be evaluated upon the arrival of the event, but since, in executable models, events are queued, guards are evaluated during the dispatching of the event (Samek, 2008, Chapter 2) (recall the sub-steps to handle an event in Section 2).

Therefore, understandability of logic-labelled finite-state machines (although completely sequential), seems also to require a certain level of maturity and familiarity with UML (as we mentioned in earlier sections, most experimental evaluations of artefacts and cost-effectiveness of the UML suggest expertise and significant familiarity are required). Our results are consistent with this. We evaluated the understandability/efficiency of the subjects as the accuracy of questions about the LLFSM terminating (or running in a continuous loop), whether the execution leaves the state named Initial without executing the Do (Internal) section, and whether the On-Exit of the NEXT state is executed because no transition fires. Therefore, a fourth element is that, when in state NEXT the Do does run. We had 21 respondents on our first campus, 10 graduate students and 11 undergraduate students. The accuracy divided by the time taken is used as understandability/efficiency and the values satisfy a normal distribution assumption with a Q-Q plot (for each group). The graduate students' mean understandability/efficiency is superior to that of the undergraduate students (statistically significant at a $\gamma=95 \%$ confidence level). Upon replication on the other campus, we had 6 undergraduate volunteers and 12 graduate volunteers. Despite the lower numbers, 
we also saw a significant result (at $\gamma=95 \%$ ), showing a superior understandability/efficiency for graduate students over the mean for undergraduate students.

\subsection{Nested LLFSMs}

We assessed the understandability/efficiency, evaluating the subjects' accuracy when the two LLFSMs (Figure 4 and Figure 5) are placed in different initial order in the execution arrangement. This swapping of the initial conditions modifies the schedule slightly, and the output varies. Moreover, in this case, the execution continues endlessly. Our results indicate a similar pattern as previously. The first campus had 14 undergraduate and 10 graduate students, the second campus had 10 undergraduate and 11 graduate respondents. Performance was significantly superior for graduate students at $\gamma=95 \%$. The undergraduate students seem to follow each LLFSM separately. But these subjects could not master the notion of ringlet (and of round-robin schedule of the concurrent execution of the two LLFSMs) with the same understandability/efficiency of the graduate students.

\subsection{Subsumption and Delegation}

All groups of students, in this case, opted for the incorrect implementation pattern. The result is that none of the subjects obtained a correct implementation with LLFSMs of the model in Figure 2.

\subsection{Randomised Diagrams}

Here we used first a 3-factor ANOVA (between subjects / one-way) analysis, as we identify the three types of diagrams. If we measure the accuracy on the 8 questions in Figure 6 divided by time, we find no evidence that the means are different.

The box-plot in Figure 7 (generated with R's ggplot (R Core Team, 2016)) shows not much difference, except for one outlier where one subject did extremely well for the LLFSM diagram using the delegation pattern. However, if we break the questionnaire into the four middle questions $(3,4,5$, and 6 , which deal with Run-Until-Completion semantics), we obtain that the results are significantly better for understandability/efficiency for diagrams with suspend/resume (refer to Figure 8).

Conversely, on Questions 1, 2, 7, and 8, the UML diagram performs much better. Again, the 3-factor ANOVA results show no statistically significant difference. However an unpaired (two sample) $t$-test of the understandability/efficiency on the UML diagram versus the suspend/resume diagram does indicate the

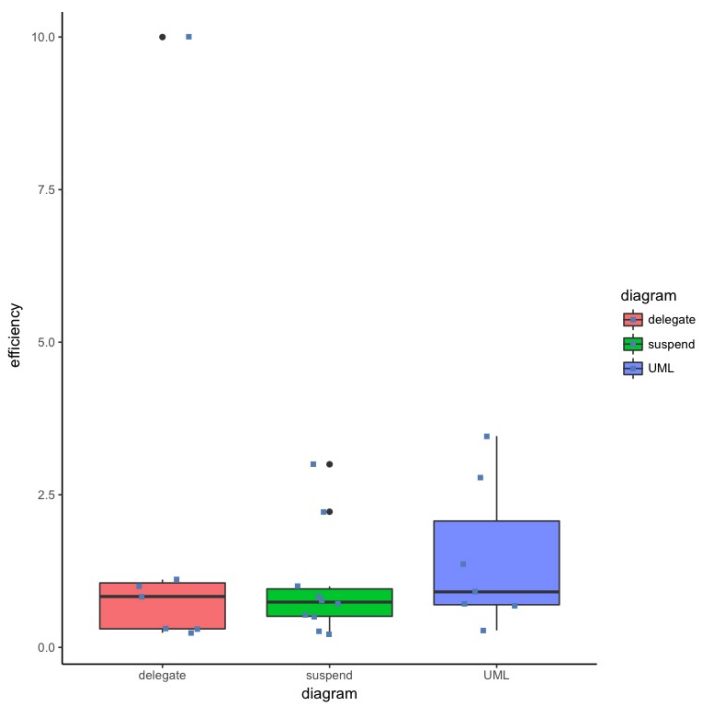

Figure 7: Box plot of the 3-ways understandability/efficiency per model type of the Figure 6 questions.

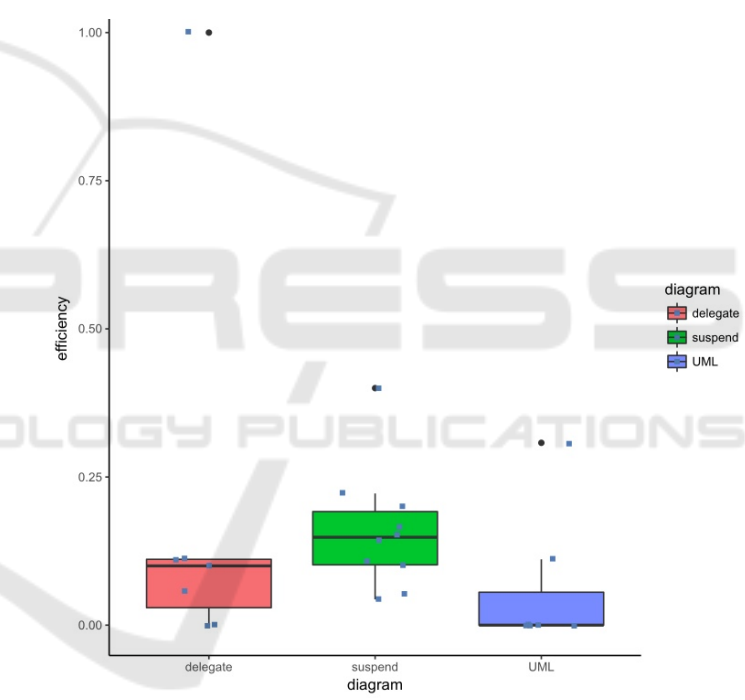

Figure 8: Box plot of the 3-ways understandability/efficiency per model type on Q's 3 to 6 for Figure 6.

rejection of the null hypothesis at $95 \%$. That suggests UML diagrams are understandable as long as we set up scenarios with well-spaced events, where users can follow all the consequences of one event before the arrival of another. LLFSM seems to be the other way around. While, at a first glance, this run-untilcompletion semantics appears obvious and straightforward, in our experimental task with Figure 2 and Figure 6, almost all subjects had substantial trouble with Questions 4 to 8. 


\section{DISCUSSION}

As in many controlled experiments, students are not equivalent to professionals in their regular software development projects. Nevertheless, for evaluating understandability, the lack of experience is potentially a catalyst for the difficulty in managing the models (Mustafa, 2010) This does, however, emphasise that performing experiments in realistic circumstances is very relevant (Dzidek et al., 2008).

Since the tasks are simple or at least not very elaborate (for example, Figure 2 and Figure 6), it is possible that in sophisticated settings the results could be different. The situation in industrial scenarios may involve complex behaviours of many interconnected state charts, staff under pressure, and specific tools for development, where understandability interacts with other factors in more complicated ways than presented here. The experiments were conducted separately, allowing at least one week in between to minimise the effect of fatigue. Plagiarism was eliminated by ensuring individuals worked without coaching, advice, or communication with others. However, using simple tasks for UML diagrams is a suitable approach when dealing with subjects that are students, as long as we keep in mind that the experience of users could have a more profound effect on complex tasks (Mustafa, 2010). Kitchenham et al. (2002) consider students suitable subjects for these scenarios. While using simple tasks does not invalidate the results here, it is possible that our results do not extrapolate to sophisticated and complicated scenarios.

Another matter of potential external validity is the suitability of the UML refresher material. Results may vary with different materials or with different delivery of such materials. We noticed these students effectively had little prior exposure to the notion of state machines. Neither of five programs feeding into our subject pool had a course on automata and formal languages, nor a course in compilers or content on lexical analysis. Therefore, results may vary if subjects had exposure to state machines in other areas of IT.

For construct validity (the suitability of the instrument to measure understandability), we faced the same challenges as all other studies: understanding is a cognitive process, we can only measure performance elements that we believe reflect the level of understanding. Failure to achieve a task (such as translation into another language or into the output behaviour sequence) is considered linked to a lack of understanding. But the possibility exists that UML notations are simply hard to learn (which may be associated with understandability). We also may not have been able to measure the understanding failures accurately.

With respect to statistical validly, challenges could be derived from violations to the statistical assumptions that enable a particular analysis, low statistical power or low effect size. In testing between two groups, we used the $t$-test. Where we employ ANOVA, we assume homogeneity of variance as there does not seem to be any other factor that would invalidate this assumption. Verifying our data using a Q-Q-plot indicated normally distributed random variables. When partitioning, each value was sampled independently from any other variable to ascertain between-subject factors. However, we acknowledge that our sample sizes were smaller than those in other, similar studies. Nevertheless, we considered results only where we could report statistical significance. The class sizes from where students were recruited were much larger than the samples reported, as participation was voluntary. This self-selection of the subjects implies a potential bias.

\section{DETERMINISTIC SEMANTICS}

We have challenged here the simplistic, initial view of our first Wikipedia quote that "Regardless of how a state is entered or exited, all its entry and exit actions will be executed" (also (Samek, 2008, Page 76)). We argue that there is an inherent asymmetry that is disregarded by such a semantics. This asymmetry is caused by the fact that suspension of a machine is a meta-action (from the perspective of the machine being suspended) that is performed by the scheduler (when triggered, for example, by a higher level machine in the subsumption architecture). That is, it would be surprising if a machine that is suspended (and thus no longer operating) were to execute its normal, operational On-Exit actions. The toaster example in Figure 2 illustrates this, because the obvious expectation is for the oven to be immediately turned off when the door is detected as open. This is seemingly at odds with the event-driven nature of UML with its associated run-until-completion semantics, that demands that all associated On-Exit actions still be performed. Not only does this put a high cognitive load on anybody trying to comprehend a corresponding set of state charts, but perhaps more importantly, this also places a high cognitive load on the designer who would have to cater for the fact that these actions need to be performed regardless of whether the machine operates normally or is being suspended. This clearly violates the principle of the least surprise and can have severe consequences in safety-critical systems (such 
as the radiation magnetron of a microwave), where two opposing concerns (normal operation and immediate shutdown) suddenly have to be catered for in the same (On-Exit) action.

Hence, there is an asymmetry of the On-Entry and On-Exit actions that is rooted in the inherent asymmetry of the execution context and sphere of control (subsystem vs meta-action), which means that the composition through sub-machines suggests that an alternative semantics is required. This alternative is the sequential execution of logic-labelled finitestate machines. In our study, we observed a significant difference in the number of subjects that have a preference to treat the implementation in Figure 2 by the suspend/restart mechanism when the DOOR_OPEN/DOOR_CLOSED (respectively) signals are detected. However, what should be the semantics of a machine that receives a suspend with respect to the On-Exit section of its current state?

The semantics of an arrangement of LLFSMs is that all machines in the arrangement are executing concurrently, but only one at a time has the token of execution. When the holder of token runs the actions associated with its current state, it executes one ringlet on the current state and then the token of execution is passed to the next machine by the scheduler. Execution of a ringlet is defined as follows.

1. If (and only if) the previous state was different to the current one, the On-Entry action is executed.

2. Transitions are evaluated in their predefined sequence, if none of them is true, the Do section is executed and the ringlet finishes.

3. If a transition evaluates to true, the On-Exit is executed, and the transition target state becomes the current state. This also completes the ringlet.

Therefore, the On-Entry section of a state is executed once and only once, without exception when arriving from another state (this issue had also had some debate for some special cases in SCXML (World Wide Web Consortium, 2005)).

However, when a machine gets suspended, it does not currently possess the token of execution. Typically, the suspend operation is triggered by another (higher level, controller) machine. This means that from the perspective of the machine being suspended, the suspend happens outside its own sphere of control at a time it does not run its ringlet. The rationale is that, at least for robotic and embedded systems, which use the suspend/resume/restart mechanisms, often derived from Brooks (1986) subsumption architecture, a suspended machine should not execute anything further past the signal that triggered the suspension. Consider for example a robot that, in a subma- chine uses an On-Entry state to arm a motion-related action that later gets triggered in On-Exit, e.g. once an object comes into vision. In this scenario, a supermachine ensures some safety constraints, for example, that the robot is in a safe, standing position. If the robot were to fall, and the super-machine were to suspend the inner machine, executing the On-Exit of the sub-machine that triggers the motion, would cause the robot to perform a motion in an unsafe posture.

In Figure 2, the higher-level machine is a switching behaviour between toasting and door_open. The inner behaviour, the submachine that switches between toasting or baking, will only receive the token of execution after it has been suspended, i.e. after the controller machine has performed the suspend. By the time the inner machine is to receive back the token of execution, it already is in the state of suspension. Therefore it would be quite surprising if it were to resume its prior state just for the purpose of executing the associated On-Exit action.

We do not challenge the beauty of the symmetry of On-Entry and On-Exit actions. Instead, we argue that this symmetry only applies while the corresponding machine is in control of its operation. In other words, this symmetry applies to a finite state machine, but not to any operations that fall outside its own sphere of control. The above illustrations serve to further our argument. The On-Exit is to be executed when one of the expressions labelling a transition in that inner machine becomes true. Executing the On-Exit on suspension means the On-Exit is executed in a completely unpredictable context (that might change with any change of composition of components), and precisely a context where none of the conditions has been met that are stated in the expressions that label the transitions leading away from the state in question; what could be more unsafe? This also explains the seeming asymmetry of the On-Entry, which does indeed get executed when the operation of a machine is resumed. Akin to when a machine is first started and the On-Entry of its initial state is executed, the corresponding On-Entry is executed when a machine that was previously suspended gets resumed or restarted. This is consistent with the fact that the corresponding machine is in control and therefore able to perform its specified actions.

The execution of On-Exit precisely, and only when one of the transitions is has its guard evaluated to true, works as a precondition (in the strictest sense of programming by contract Mitchell et al. (2002)). This also enables a locality of concerns for developers, which can genuinely develop in the subsumption architecture, where lower layers are completely unaware of higher layers. This is one of the most impor- 
tant principles facilitating code re-use and this separation of concerns and dependency on higher layers loosens coupling. Therefore, our semantics enforce a stronger, first-principles based method of software development with state chart models.

Our alternative semantics does not prevent the implementation of UML nested states. For example, in Figure 2 we have the situation where the DOOR_OPEN signal acts as a trigger to several On-Exit actions (in the outer and in the inner machines), and where such actions ensure switching off or putting on hold aspects that were enacted or turned on in the corresponding On-Entry. In this case, suspending the inner machine and not executing the On-Exit would leave the toaster on. The point is that when a super-machine requires cooperation from a sub-machine this should be made explicit through notification (to the sub-machine) of a condition. Software patterns that should be suitable here would be delegation and forwarding of a corresponding signal. Moreover, using delegation makes the order in which all the nested states execute their corresponding On-Exit actions very explicit. Note, however, that the original description by Harel prioritised first the super-state over the sub-state, while later, the UML used an inverse prioritisation. This, once more, emphasises the significance of clear, accessible semantics to the designer. Therefore, we can see the importance of the discussion here to characterise precisely the situations where the subsumption architectural pattern of independent components is applicable, versus those situations where other patterns, such as delegation and communication, are applicable. Making these explicit to software engineers may alleviate the confusion that exists, as our experiments have revealed.

\section{CONCLUSIONS}

One of the most surprising findings from our perspective is the remarkable belief in the population of subjects that UML state charts imply strong restrictions on the order of events. For example, with respect to Figure 2, the $32 \%$ of the first-campus group (52 respondents) indicated in one particular question that the diagram implies that DOOR_OPEN would always happen before DOOR_CLOSED (the group on the second campus had 26 respondents but $38 \%$ also believed such ordering of events). This is despite demonstrations prior to the questionnaire with tools like $Q M^{\mathrm{TM}}$ that in these type of diagrams, all sequences of events of the form

$$
\text { (DOOR_CLOSED|DOOR_OPEN)* }
$$

are valid and that the behaviour would just toggle between door_open and heating at the right time. That is, duplication of DOOR_OPEN once in the state door_open has no effect. For the same set of subjects, $68 \%$ could not commit either way about whether the diagram implied something regarding the order of events. Only $10 \%$ could confirm that the UML diagram in Figure 2 is at the mercy of whatever sequence of events, and should be designed so that it behaves correctly and should not expect an environment that plays benevolently.

There is significant overlap within the UML with respect to state diagrams and activity diagrams. Samek (2008) already dedicates a section to the commonalities and distinctions between UML state charts and activity diagrams. The data-flow or control flow of activity diagrams seems to muddle the understanding of UML's event-driven nature.

Clearly, logic-labelled finite-state machines, although apparently simpler (they seem like UML state charts without events), constitute a much more precise and unambiguous semantics that provides complete detail for execution. As such, they seem to require significantly more maturity from users. However, when issues of timing and order of execution become more critical, or when interpreting and understanding the effect of a shower of events, or the handling of events while another event is still being operated, LLFSMs are much clearer and transparent.

The UML's treatment of On-Exit and On-Entry sections aims for absolute symmetry. However, such symmetry is not reflected by experimental evaluation of user understandability/efficiency of UML state charts. Moreover, in the case of executable models, such as LLFSMs, and in the context of robotic and embedded systems, it is extremely important to delineate the precise semantics derived from such asymmetry. We have argued for a semantics where On-Exit is executed upon leaving the state in the sphere of control of the current machine.

\section{REFERENCES}

Basili, V. R., Shull, F., and Lanubile, F. (1999). Building knowledge through families of experiments. IEEE T. on Software Engineering, 25(4):456-473.

Börger, E., Cavarra, A., and Riccobene, E. (2000). Modeling the dynamics of UML state machines. In $A b$ stract State Machines-Theory and Applications, pages 223-241. Springer.

Brooks, R. (1986). A robust layered control system for a mobile robot. IEEE J. of Robotics and Automation, 2(1):14-23.

Cruz-Lemus, J. A., Genero, M., Manso, M. E., Morasca, S., 
and Piattini, M. (2009). Assessing the understandability of UML statechart diagrams with composite statesa family of empirical studies. Empirical Software Engineering, 14(6):685-719.

Cruz-Lemus, J. A., Genero, M., Manso, M. E., and Piattini, M. (2005). Evaluating the effect of composite states on the understandability of UML statechart diagrams. Model Driven Engineering Languages and Systems, pages 113-125, Berlin, Heidelberg. Springer.

De Lucia, A., Gravino, C., Oliveto, R., and Tortora, G. (2010). An experimental comparison of ER and UML class diagrams for data modelling. Empirical Software Engineering, 15(5):455-492.

Douglass, B. P. (1999). Doing Hard Time: Developing Real-time Systems with UML, Objects, Frameworks, and Patterns. Addison-Wesley, Reading, MA.

Drusinsky, D. (2006). Modeling and Verification Using UML Statecharts: A Working Guide to Reactive System Design, Runtime Monitoring and Execution-based Model Checking. Newnes.

Dzidek, W. J., Arisholm, E., and Briand, L. C. (2008). A realistic empirical evaluation of the costs and benefits of UML in software maintenance. IEEE Trans. Softw. Eng., 34(3):407-432.

Estivill-Castro, V., Hexel, R., and Rosenblueth, D. A. (2012). Efficient model checking and FMEA analysis with deterministic scheduling of transition-labeled finitestate machines. 3rd World Congress on Software Engineering (WCSE 2012), pages 65-72, Wuhan, China. IEEE Computer Soc. (CPS).

Genero, M., Miranda, D., and Piattini, M. (2003). Defining metrics for UML statechart diagrams in a methodological way. Conceptual Modeling for Novel Application Domains, pages 118-128, Berlin, Heidelberg. Springer.

Glinz, M. (2000). Problems and deficiencies of UML as a requirements specification language. Proc. 10th Int. Workshop on Software Specification and Design, page 11. IEEE Computer Soc.

Harel, D. and Politi, M. (1998). Modeling Reactive Systems with Statecharts: The STATEMATE Approach. McGrawHill, New York, NY.

Höst, M., Regnell, B., and Wohlin, C. (2000). Using students as subjects - a comparative study of students and professionals in lead-time impact assessment. Empirical Software Engineering, 5(3):201-214.

Kitchenham, B. A., Pfleeger, S. L., Pickard, L. M., Jones, P. W., Hoaglin, D. C., El Emam, K., and Rosenberg, J. (2002). Preliminary guidelines for empirical research in software engineering. IEEE T. on Software Engineering, 28(8):721-734.

Liskov, B. H. and Wing, J. M. (1994). A behavioral notion of subtyping. ACM Trans. Program. Lang. Syst., 16(6):1811-1841.

Mellor, S. J. (2000). UML point/counterpoint: Modeling complex behavior simply. Embedded Systems Programming.

Mitchell, R., McKim, J., and Meyer, B. (2002). Design by Contract, by Example. Addison-Wesley, Reading, MA.
Mustafa, B. A. (2010). An experimental comparison of use case models understanding by novice and high knowledge users. New Trends in Software Methodologies, Tools and Techniques - Proceedings of the 9th SoMeT_10, volume 217 of Frontiers in Artificial Intelligence and Applications, pages 182-199. IOS Press.

Object Management Group (2017). Action language for foundational UML (alf) - concrete syntax for a uml action language. Version 1.1. Technical Report formal/2017-07-04, An OMG Action Language for Foundational UML Publication, The address of the publisher. Normative reference: http://www.omg.org/ spec/ALF/1.1.

Petre, M. (2013). UML in practice. Proc. 2013 Int. Conf. on Software Engineering, ICSE '13, pages 722-731, Piscataway, NJ, USA. IEEE Press.

R Core Team (2016). R: A Language and Environment for Statistical Computing. R Foundation for Statistical Computing, Vienna, Austria.

Reggio, G., Astesiano, E., Choppy, C., and Hussmann, H. (2000). Analysing UML active classes and associated state machines-a lightweight formal approach. In Fundamental Approaches to Software Engineering, pages 127146. Springer

Reggio, G., Leotta, M., Ricca, F., and Clerissi, D. (2013). What are the used UML diagrams? a preliminary survey. Proc. of 3rd Int. Workshop on Experiences and Empirical Studies in Software Modeling (EESSMod co-located with MODELS, volume 1078, pages 3-12. CEUR.

Robbins, J. e. (1999). Cognitive Support Features for Software Development Tools. PhD thesis, Department of Information and Computer Science, University of California, Irvine. Advisor: Prof. D. F. Redmiles.

Rumpe, R. (2002). Executable modeling with UML - a vision or a nightmare? -. Issues and Trends of Information Technology Management in Contemporary Associations Volume 1, pages 697-701. Idea Group Publishing.

Samek, M. (2008). Practical UML Statecharts in $C / C++$, Second Edition: Event-Driven Programming for Embedded Systems. Newnes, Newton, MA, USA.

Wood, S. K., Akehurst, D. H., Uzenkov, O., Howells, W. G. J., and McDonald-Maier, K. D. (2008). A modeldriven development approach to mapping UML state diagrams to synthesizable VHDL. IEEE T. on Computers, 57(10):1357-1371.

World Wide Web Consortium (2005). State chart XML (SCXML): State machine notation for control abstraction. 